\title{
Thiol-functionalized silica colloids, grains, and membranes for irreversible adsorption of metal(oxide) nanoparticles
}

\author{
E. Maria Claesson*, Albert P. Philipse \\ Van't Hoff Laboratory for Physical and Colloid Chemistry, Debye Institute, Utrecht University, \\ Padualaan 8, 3584 CH Utrecht, The Netherlands \\ Received 23 January 2006; received in revised form 10 August 2006; accepted 9 October 2006 \\ Available online 13 October 2006
}

\begin{abstract}
Thiol-functionalization is described for silica surfaces from diverging origin, including commercial silica nanoparticles and Stöber silica as well as silica structures provided by porous glasses and novel polymer-templated silica membranes. The functionalization allows in all cases for the irreversible binding of metal(oxide) particles from a solution. Examples are the adsorption of $\mathrm{CoFe}_{2} \mathrm{O}_{4}$ particles for the preparation of magnetizable silica colloids and silica structures, and gold nanoparticles that directly nucleate and grow on thiol-functionalized silica in a solution of $\mathrm{HAuCl}_{4}$ upon addition of a reducing agent.
\end{abstract}

(C) 2006 Elsevier B.V. All rights reserved.

Keywords: Gold particles; Silica colloids; Silica-metal composites; Thiol-functionalization; Polyethersulfone membranes

\section{Introduction}

For many technical applications, it is desirable to chemically modify the surface of nanostructured materials, such as colloidal particles, porous micron sized grains and membranes [1-4]. Such materials provide large surface-to-volume ratios and at the same time they can readily be separated from a dispersion medium either by sedimentation, removal by a membrane or when having magnetic properties - with an external magnetic force, making them suitable for separation purposes. Arakaki et al. for instance have reported on thiol-functionalized silica gel as adsorbent to remove toxic metal species, such as mercury from aqueous solutions [5]. Moreover, chemical adsorption of metals to particles and grains might also be interesting for applications in for instance decorative coatings due to their color properties.

A large variety of nanostructured materials are made of, or can be coated with silica [6-12]. An important advantage of silica is its well-known surface chemistry and the broad spectrum of organosilanes available to modify the silica surface with functional groups to give it desired properties $[13,14]$. This type of

\footnotetext{
* Corresponding author. Tel.: +31 30253 5990; fax: +31 302533870 .

E-mail address: e.m.claesson@ chem.uu.nl (E.M. Claesson).

$U R L$ : www.chem.uu.nl/fcc.
}

modification has for instance been used to stabilize colloidal silica in organic solvents [14] but also to chemically bind magnetic metal oxide particles [15] or other metal nanoparticles to silica particles $[4,16-18]$ to give them different magnetic or optical properties.

In this work, we have investigated functionalization of a variety of silica nanostructures with thiol-groups as a general method for chemical binding of various metal or metal oxide particles to silica. Gold particles were used as a probe for the presence of thiol surface groups since it is well-known that gold has a high affinity for thiols [18-20]. These gold particles, synthesized by reduction of gold chloride hydrate $\left(\mathrm{HAuCl}_{4}\right)$ with sodium borohydride $\left(\mathrm{NaBH}_{4}\right)$ are small $(R \sim 2 \mathrm{~nm})$ and have a high electron density which makes them easy to distinguish from the silica during electron microscopy characterization. The following four types of silica structures were investigated:

(1) Commercially available small silica particles (Bindzil, $R \sim$ $6 \mathrm{~nm}$ ), for which thiol-functionalization and metal adsorption, as far as we know, have only been described for gelated particles [21] but not for silica particles of this size while they remain in a stable dispersion.

(2) Larger silica colloids prepared with the Stöber method $[6,22](R=158 \mathrm{~nm})$, as well as magnetic Stöber silica with embedded cobalt ferrite $\left(\mathrm{CoFe}_{2} \mathrm{O}_{4}\right)(R=217 \mathrm{~nm})[15]$. 
(3) Micron-sized porous silica grains, such as so-called CPG's (controlled porous glasses, see for instance refs. [8,9]) and the mesoporous silica structure SBA-15 [7].

(4) Commercially available polyethersulfone (PES) filter membranes were coated with the same method, after that these membranes had first been embedded in a silica crust.

Polymeric materials cannot as easily be chemically modified as silica, but it was recently shown that PES membranes can be encapsulated in silica by performing Stöber synthesis directly onto the polymer [23]. Here, we show that the addition of Bindzil silica nanoparticles results in a smooth, complete coverage of the membranes leaving a large silica surface available for functionalization with organosilanes.

The size, shape, and gold particle coverage of the various silica particles were characterized with transmission- and scanning electron microscopy. The presence of thiol-groups on the smallest silica particles was additionally investigated with infrared spectroscopy.

\section{Experimental}

\subsection{Materials}

Tetraethoxysilane (TES, Fluka), ethanol (technical grade), ammonium solution (28.6\%, Merck), gold chloride hydrate (Fluka), 3-mercaptopropyl trimethoxysilane (MPTMS, 97\%, Fluka), sodium borohydride (Aldrich). Commercial silica dispersion $\left(\right.$ Bindzil $^{\circledR}$ 30/360, Eka Chemicals $A B$ ), controlled porous glass (CPG0300C, Controlled Pore Glass Inc.) $\mathrm{AG}^{\circledR}$ 501-X8 and Bio-Rex ${ }^{\circledR}$ MSZ 501 (D) mixed bed resin (Bio Rad), Millipore Express ${ }^{\circledR}$ PLUS Membrane, polyethersulfone, hydrophilic, $0.22 \mu \mathrm{m}, 47 \mathrm{~mm}$ (Millipore Corporation). For synthesis of silica particles, the ethanol and TES were distilled prior to use. In all other cases, the chemicals were used as received. A stock solution of $100 \mathrm{~mL}$ of approximately $0.02 \mathrm{M} \mathrm{HAuCl}_{4}$, assuming $\mathrm{HAuCl}_{4} \cdot 6 \mathrm{H}_{2} \mathrm{O}$, was prepared by dissolving $0.90 \mathrm{~g}$ $\mathrm{HAuCl}_{4}$ (Fluka) in doubly distilled water. This stock solution of $\mathrm{HAuCl}_{4}$ was used throughout all experiments. The sodium borohydride solutions were prepared less than $30 \mathrm{~min}$ before each experiment due to the rapid oxidation of $\mathrm{NaBH}_{4}$ in water.

\subsection{Thiol-functionalization and gold particle synthesis onto Bindzil silica nanoparticles}

\subsubsection{Surface modification of Bindzil with \\ 3-mercaptopropyl trimethoxysilane}

Bindzil silica nanoparticles were received as alkaline ( $\mathrm{pH} 10)$ aqueous dispersions, and had to be transferred to ethanol for the MPTMS grafting procedure. However, the dispersion contains soluble silicates that precipitate and cause gelation when adding ethanol. Therefore, the first step was to remove these silicates by adding $10 \mathrm{~g}$ of a AG501-X8 and Bio-Rex MSZ 501 (D) mixed bed ion exchange resin (Bio Rad) in $100 \mathrm{~mL}$ Bindzil-dispersion diluted to $200 \mathrm{ml}$ with doubly distilled water. The resin was left in the dispersion for several weeks [24]. After 2 weeks, the $\mathrm{pH}$ had decreased from 10 to 7 , indicating all ions initially present in the solution had been changed to $\mathrm{H}^{+}$or $\mathrm{OH}^{-}$. The resin was filtered out, and the dispersion was dialyzed for 1 week against ethanol in Spectra/Por Type F polyvinylidene difluoride (PVDF) dialysis membranes, refreshing the ethanol reservoir once per day. The hydrodynamic radius, $R_{\mathrm{h}}=9.5$ of the particles was determined by dynamic light scattering. This hydrodynamic radius corresponds to a number averaged radius, $R$, of approximately $6 \mathrm{~nm}$, for a polydispersity of $35 \%$ [25].

To a mixture of $10 \mathrm{~mL}$ of the Bindzil dispersion in ethanol $\left(\phi_{\mathrm{m}}=0.167 \mathrm{~g} / \mathrm{mL}\right), 1 \mathrm{~mL} \mathrm{NH} 3$ and $90 \mathrm{~mL}$ ethanol $(\mathrm{pH} 8.5)$, $5 \mathrm{~mL}$ MPTMS was added and the mixture was stirred for $40 \mathrm{~min}$ before heating it to $\sim 80^{\circ} \mathrm{C}$. Slowly, approximately $100 \mathrm{~mL}$ of ethanol and ammonia was removed by distillation at ambient pressure. Tests with various concentrations of ammonia showed that higher ammonia concentrations $(\mathrm{pH}>8.5)$ caused the Bindzil dispersion to gel. The reaction mixture was purified from unreacted MPTMS via dialysis for 2 days against ethanol. After these 2 days and after refreshing the ethanol four times, the strong, characteristic smell of MPTMS was gone. The thiolfunctionalized Bindzil particles were transferred back to (doubly distilled) water by transferring the dialysis tubes in to a water reservoir. Due to the difference in density between ethanol and water, the tubes were floating at the beginning of the dialysis and slowly sinking as the ethanol content inside the tubes was reduced. The dialysis was continued for 1 week, refreshing the water reservoir twice per day. The dialysis was stopped when the refractive index of the reservoir was the same as that of pure doubly distilled water. The resulting aqueous dispersion of $9.5 \mathrm{mg} / \mathrm{mL}$ functionalized Bindzil particles was labeled Bindzil$\mathrm{SH}$.

\subsubsection{Synthesis of gold particles onto Bindzil particles}

The gold-silica particles were prepared by reducing $\mathrm{HAuCl}_{4}$ in the presence of MPTMS-coated silica spheres. Solutions $(0.1 \mathrm{M})$ of the reducing agent, $\mathrm{NaBH}_{4}$, were prepared less than 30 min before each synthesis. In the gold particle synthesis step, $\mathrm{HAuCl}_{4}$ stock solution was added first, followed by an equal volume of $\mathrm{NaBH}_{4}$-solution. Immediately after adding the reducing agent, gas bubbles due to evolving $\mathrm{H}_{2}(\mathrm{~g})$ and a change in color, typically pink to purple, could be observed in the reaction mixture. The experiment was repeated for a certain amount of Bindzil-SH dispersion $\left(2 \mathrm{~mL}, \phi_{\mathrm{m}}=0.01 \mathrm{~g} / \mathrm{mL}\right.$, in $50 \mathrm{~mL}$ doubly distilled water) with various amounts of $\mathrm{HAuCl}_{4}$ and reducing agent. Also the effect of stirring and the $\mathrm{NaBH}_{4}$ concentration on the final product was investigated. For comparison, a blank sample was prepared using Bindzil which had not been treated with MPTMS but adding the same amount of reagents for the synthesis of gold.

\subsection{Thiol-functionalization and gold adsorption onto PES-membranes covered with a silica crust}

To provide the filter membranes with a silica layer for further functionalization, two approaches were tested. In the first method, amorphous silica was synthesized directly onto the membranes. The PES-membranes were soaked in an ammonia solution $(10 \mathrm{~mL}, 25 \%$ in $40 \mathrm{~mL}$ ethanol) for approximately 
$1 \mathrm{~h}$ before placing them in between two filter papers soaked in TEOS (10 vol\% in ethanol) [23]. To allow the reagents to be homogeneously absorbed, the PES-membranes were carefully blotted, and left for $4 \mathrm{~h}$ in a covered Petri dish. After that, the membranes were put into pure ethanol for at least 1 day to remove any free silica particles. The membranes prepared in this way were labeled PESSt. In a second approach, it was investigated whether the Bindzil particles could be adsorbed directly onto the membranes and in this way create a silica crust on the polymer. The PES membranes were left overnight in dispersions of Bindzil particles in water (label: PESBiW) as well as in ethanol (label: PESBiEt). Additionally, some membranes were pre-treated by soaking these in an ammonia solution to investigate if the attraction between the polymer and silica particles could be due to electrostatic attraction between the silica and the PES-membrane (label: PESBiEt2).

After rinsing the presumably silica covered membranes carefully, they were transferred to a mixture of $120 \mathrm{~mL}$ ethanol and $1 \mathrm{~mL} 25 \%$ ammonia solution. Under slow agitation, $0.4 \mathrm{~mL}$ (2.1 mmol) MPTMS was added and the mixture was heated to $50{ }^{\circ} \mathrm{C}$ for $15 \mathrm{~h}$. Subsequently, they were rinsed with ethanol and immersed in deionized water. We noted that the PESSt and PESBiEt2-membranes had clearly become much more hydrophilic after the treatment with MPTMS compared to the pure PES membranes. When placing water droplets on the surface with a pipet, the water was immediately absorbed, although at some parts of the PESSt membrane water stayed longer on the surface.

The functionalized membranes were left overnight in an aqueous dispersion of gold nanoparticles, prepared in the same way as described in Section 2.2.2.

\subsection{Thiol-functionalization and gold particle synthesis onto Stöber silica}

A stock-solution of silica particles $\left(R_{\mathrm{TEM}}=158 \mathrm{~nm}\right)$ was prepared by rapidly adding freshly distilled tetraethoxysilane (TES) to a mixture of $152 \mathrm{~mL}$ ammonia solution diluted to $25 \%$ and $1770 \mathrm{~mL}$ distilled ethanol according to the method of Stöber et al. [6]. Four hundred milliliters of the obtained silica dispersion was diluted to $600 \mathrm{~mL}$ with ethanol. $2.8 \mathrm{~mL}$ 3-mercaptopropyl trimethoxysilane was added and the mixture was stirred for $40 \mathrm{~min}$. Subsequently, the reaction mixture was heated to $80^{\circ} \mathrm{C}$ and the total volume was reduced to approximately two-thirds by distillation of ethanol and ammonia at ambient pressure $[15,14]$. The amount of MPTMS was chosen to be $2.6 \times 10^{-4} \mathrm{~mol} / \mathrm{m}^{2}$ silica surface [15], assuming a density of $1.8 \mathrm{~g} / \mathrm{cm}^{3}$ for the silica particles. The mixture was left to cool down to room temperature and was subsequently washed three times with $150 \mathrm{~mL}$ ethanol (by centrifugation at $513 \times g$ ) to remove any unreacted MPTMS. The thiol-functionalized particles were stored in ethanol to prevent hydrolysis of the MPTMSsilica bond.

Gold nanoparticles were synthesized in the presence of the thiol-functionalized silica particles following the same procedure as described for the Bindzil particles in Section 2.2.2, using

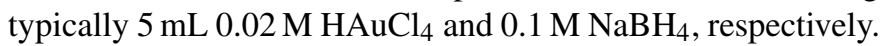

One day before an experiment, the functionalized silica particles were transferred to doubly distilled water by centrifugation and redispersion. In the gold nanoparticle synthesis, $\mathrm{HAuCl}_{4}$ solution was added first, followed by an equal volume of $\mathrm{NaBH}_{4}$ solution. All reaction mixtures were centrifuged at $228 \times g$ to remove free gold particles. The sedimentation and redispersion procedure was repeated until the supernatant had become colorless.

The silica colloids covered with small gold particles were without any further modification - coated with an outer shell of silica in a seeded growth step. Three milliliters $(0.018 \mathrm{~g})$ of the silica-gold dispersion $\operatorname{Sg} 41\left(\phi_{\mathrm{m}}=0.006 \mathrm{~g} / \mathrm{ml}\right)$ in water was added to a mixture of $5 \mathrm{~mL}$ ammonia solution and $95 \mathrm{~mL}$ ethanol and stirred vigorously while adding $0.01 \mathrm{~mL}$ TES. The reaction mixture was stirred for $4 \mathrm{~h}$. The experiment was repeated using $1.0 \mathrm{~mL}$ TES for a thicker silica layer.

\subsection{Thiol-functionalization and gold particle synthesis on magnetic silica particles}

MPTMS-functionalized silica particles were covered with magnetic cobalt ferrite particles and subsequently coated with an outer layer of silica according to ref. [15]. After purification and transfer of the thiol-functionalized particles to demineralized water, $5 \mathrm{~mL}$ cobalt ferrite dispersion was added and the mixture was gently shaken overnight. Free cobalt ferrite particles were removed by repeated sedimentation and redispersion and $30 \mathrm{~mL}$ of cobalt ferrite-silica dispersion $(0.04 \mathrm{~g} / \mathrm{mL})$ was transferred to $920 \mathrm{~mL}$ ethanol and $50 \mathrm{~mL}$ ammonia solution. Small portions of TES were added every $15 \mathrm{~min}$ to obtain a silica layer around the magnetic particles. The coated particles were sedimented by centrifugation $1 \mathrm{~h}$ at $100 \mathrm{~g}$ and redispersed three times in technical ethanol to remove any free cobalt ferrite or newly formed silica particles. The dispersion was labeled SibCs.

In the following step, $30 \mathrm{~mL}(0.002 \mathrm{~g} / \mathrm{mL})$ of the SibCs particles $\left(R_{\mathrm{TEM}}=217 \mathrm{~nm}\right)$, was diluted with $10 \mathrm{~mL}$ of ethanol, mixed with $5 \mathrm{~mL}$ ammonia solution and $0.5 \mathrm{~mL}$ MPTMS in a round bottom flask and stirred for $40 \mathrm{~min}$. Subsequently, $20 \mathrm{~mL}$ of the solvent was removed by distillation. The functionalized magnetic silica particles were purified from unreacted MPTMS by repeated centrifugation $1 \mathrm{~h}$ at $57 \times g$. The sediment was redispersed three times in ethanol and three times in doubly distilled water, resulting in a stable light brown dispersion. While gently shaking, $0.4 \mathrm{~mL} \mathrm{HAuCl}$ stock solution and $0.4 \mathrm{~mL} 0.1 \mathrm{M}$ $\mathrm{NaBH}_{4}$ was added to the thiol-functionalized magnetic silica dispersion, giving the reaction mixture a dark brown color. The particles were centrifuged $1 \mathrm{~h}$ at $57 \times g$ and the red supernatant containing excess of gold particles was removed. Due to the initial brown color of the magnetic silica, no clear difference could be observed in the color of the sediment compared to the starting dispersion.

\subsection{Thiol-functionalization and gold particle synthesis on porous silica grains}

Silica grains of micron-size porous glasses (see Fig. 8a) with a mean pore size of $367 \mathrm{~nm}$, and a surface area of $7.1 \mathrm{~m}^{2} / \mathrm{g}$ 
(data provided by supplier) were first coated with thiol-groups following the same procedure as described for Stöber silica. Three grams of CPG0300C was added to a mixture of $90 \mathrm{~mL}$ ethanol and $10 \mathrm{~mL}$ ammonia solution. To this mixture, $1.04 \mathrm{~mL}$ (5.5 mmol) 3-mercaptopropyl trimethoxysilane was added and the mixture was stirred magnetically for $40 \mathrm{~min}$, before removing approximately one-third of the ethanol and ammonia by distillation. The remaining white sediment was rinsed several times with pure ethanol to remove unreacted 3-mercaptopropyl trimethoxysilane. Subsequently, the CPG-grains were transferred to doubly distilled water and $2.5 \mathrm{~mL}$ of the $0.02 \mathrm{M}$ $\mathrm{HAuCl}_{4}$-solution was added while stirring. After $10 \mathrm{~min}$, the stirring rate was increased and $2.5 \mathrm{~mL} 0.1 \mathrm{M} \mathrm{NaBH}_{4}$ was added, upon which the color turned purple. The grains were subsequently sedimented and rinsed several times with doubly distilled water until the supernatant became colorless and transparent. The color of the resulting sediment was light brown. The synthesis of gold nanoparticles was repeated - with the same amounts - for $3 \mathrm{~g}$ CPG0300C which had not been coated with MPTMS. After rinsing the sediment with water, it had regained its original white color.

The procedure was repeated for SBA-15 mesoporous silica (see Fig. 8c). Also this resulted in a pink-colored sediment, which remained colored after several rinsing steps.

\subsection{Characterization techniques}

Transmission electron microscopy was performed on a Philips tecnai 12 microscope operating at $120 \mathrm{kV}$. TEM-samples were prepared by depositing drops of diluted dispersion on formfar-coated copper grids. Scanning electron microscopy was carried out on a Philips SEMXL30SFEG microscope operating at $30 \mathrm{kV}$. Dynamic light scattering was performed on a homebuilt set-up measuring at $\lambda=514.5 \mathrm{~nm}$. Infrared spectroscopy was performed on a Perkin-Elmer System 2000 FT-IR spectrometer. Since MPTMS is present only at the surface of the silica particles, the MPTMS volume content in relation to $\mathrm{SiO}_{2}$ is low. The samples were therefore measured on a self-supporting pellet, consisting of compressed silica particles only. Additionally, the sample was heated to $120^{\circ} \mathrm{C}$ under vacuum while measuring to remove water and thereby reducing the $\mathrm{O}-\mathrm{H}$ peak in the spectrum. In this way, a higher concentration of sample is measured, with the drawback of overload of the signals from silica. However, since the peak of interest, the $\mathrm{S}-\mathrm{H}$ stretch peak does not overlap with signals detected from silica, this is not a problem.

\section{Results and discussion}

\subsection{Thiol-functionalization and gold particle synthesis on Bindzil particles}

The experiments with the Bindzil dispersion, show that the procedure for grafting Stöber silica particles with MPTMS, as previously described in literature $[14,15]$, can be applied also to commercial aqueous silica sols. However, the very small particle size and the presence of soluble silicates in the aqueous phase required a few modifications of the thiol grafting proce-

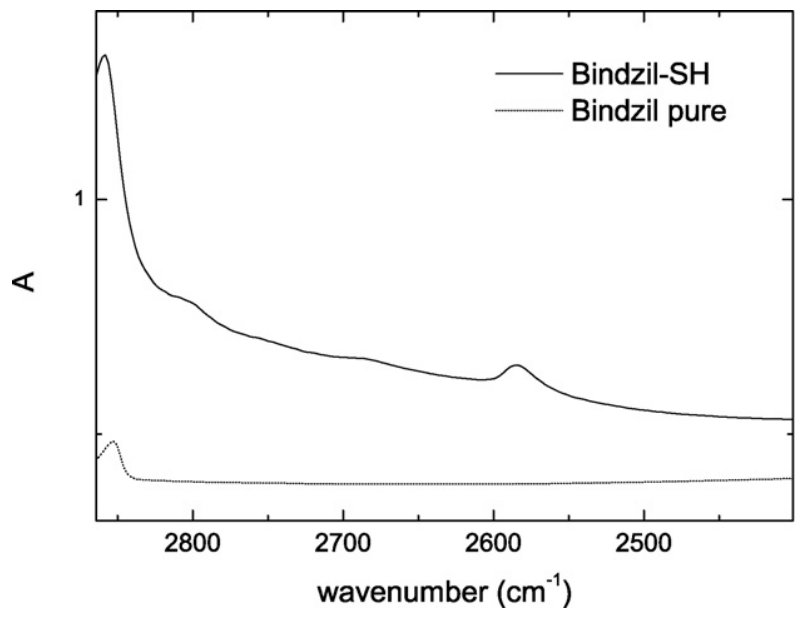

Fig. 1. IR-spectra of Bindzil particles before and after thiol-functionalization, respectively. In the spectrum of thiol-functionalized (Bindzil-SH), a weak, but clear $\mathrm{S}-\mathrm{H}$ stretch band can be found around $v=2585 \mathrm{~cm}^{-1}$, which cannot be observed in the spectrum of the pure Bindzil. Since the IR-spectra were made after thorough purification from free MPTMS, it can be concluded that the MPTMS is indeed bound to the silica particles.

dure: firstly, the silica sol as received had to be purified from soluble silicates with a mixed bed ion-exchange resin to avoid gelation when adding ethanol. After treatment with the resin, the small silica particles could be transferred to ethanol without gelation. Secondly, to avoid centrifugation at high speed for a long time, which would have been required to sediment particles of this size, dialysis was employed to change the solvent from water to ethanol and vice versa when necessary. Finally, the $\mathrm{pH}$ of the ethanol/ammonia mixture should not be higher than 8.5 to avoid gelation. This repeatedly observed gelation is not likely due to an increase in ionic strength upon adding ammonia. This follows from the observation that even a concentration of $10 \mathrm{mM} \mathrm{LiNO}_{3}$ in the Bindzil dispersion does not produce a gel. Possibly too much ammonia promotes condensation of surface silanol groups from different particles. Note in this respect that the number concentration of the small Bindzil particles is very high. After treatment with MPTMS, the Bindzil particles were stable in water as well as in ethanol. FT-IR spectra of Bindzil particles before and after treatment with MPTMS are shown in Fig. 1. In the spectrum of Bindzil-SH a weak, but clear $\mathrm{S}-\mathrm{H}$ stretch band can be found around $v=2585 \mathrm{~cm}^{-1}$, which cannot be observed in the spectrum of the pure Bindzil. Since the IR-spectra were made after thorough purification from free MPTMS, it can be concluded that the MPTMS is indeed chemically bound to the silica particles.

After performing the reduction of $\mathrm{HAuCl}_{4}$ in the presence of MPTMS-grafted Bindzil particles, the resulting brightly redcolored dispersions were stable without any gelation or flocculation for at least 3 months. Unfortunately, the $6 \mathrm{~nm}$ silica particles melt in the electron beam when performing TEM (see Fig. 2b) so that direct confirmation of that gold particles are attached to the silica particles is hindered. Nevertheless, several observations clearly confirm the (covalent) attachment of gold particles to the Bindzil particles: without the MPTMS present, the gold particles coalesce and large gold clusters can be found on the silica melt (see Fig. 2a). When using MPTMS- 
(a)

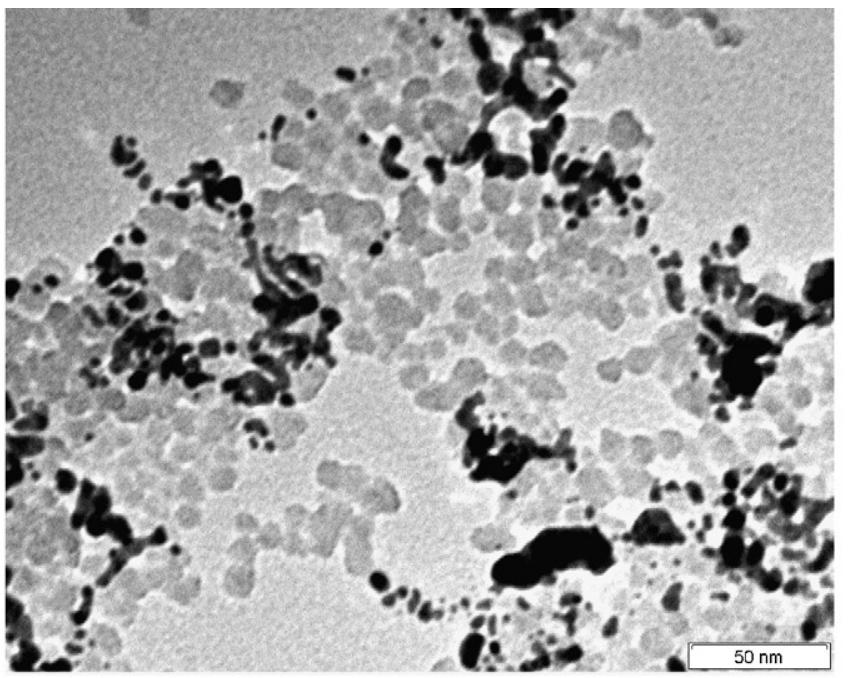

(c)

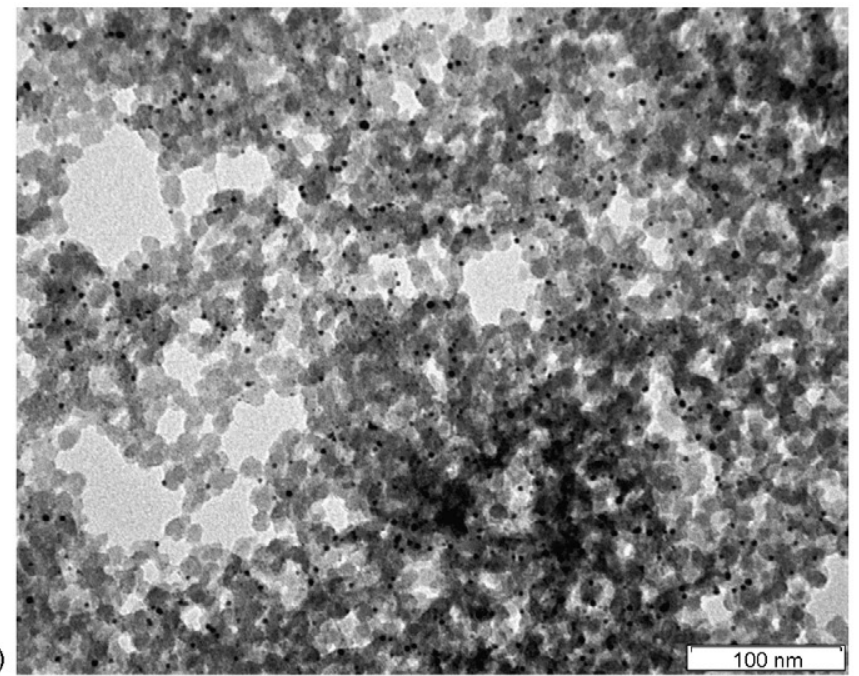

(b)

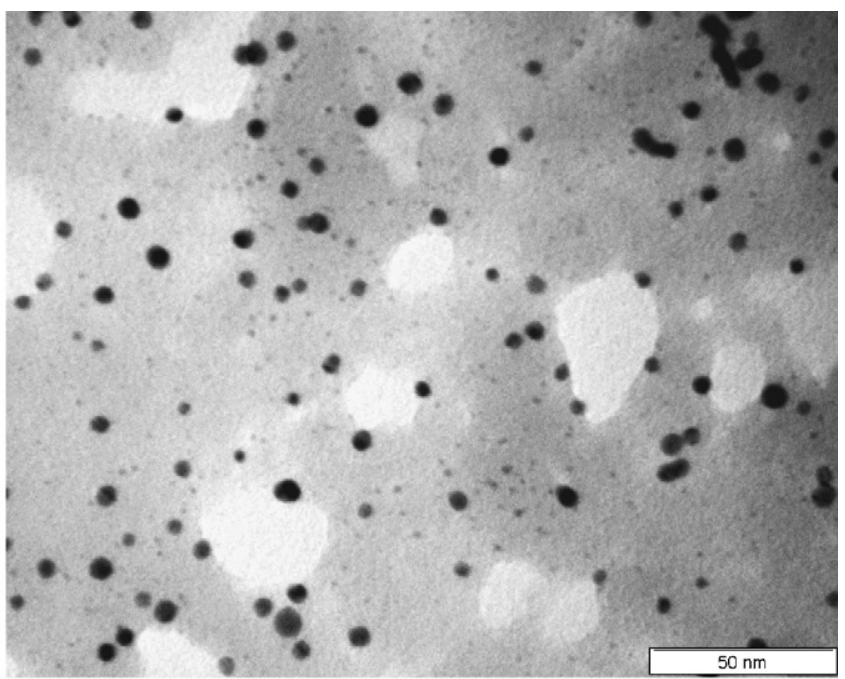

(d)

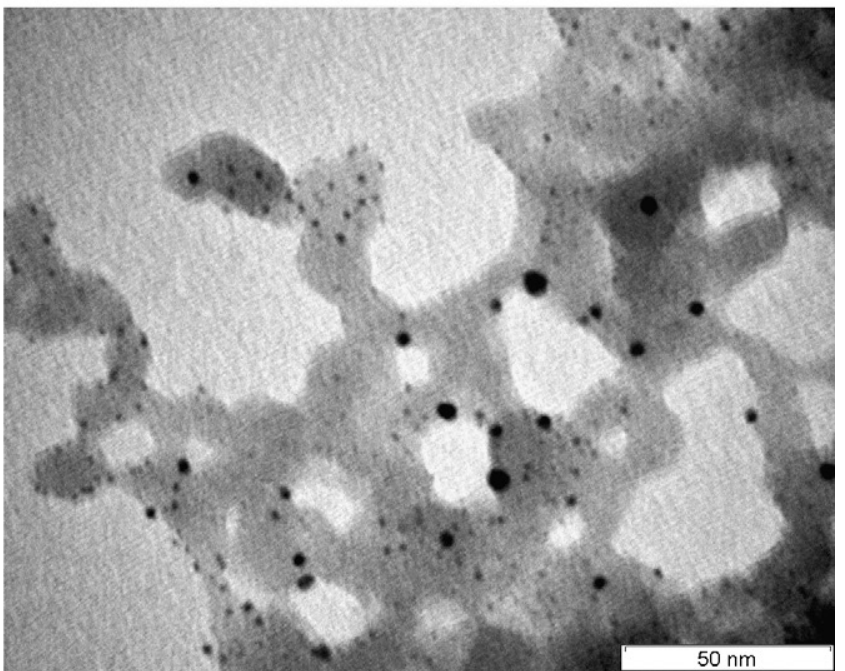

Fig. 2. TEM pictures showing the results of direct synthesis of gold particles in the presence of Bindzil particles. Although the silica particles are melting in the electron beam of the microscope, it can be observed that the sample where MPTMS is present discrete gold nanoparticles are surrounding the particles, whereas the gold particles in the sample where no MPTMS had been added, the gold particles coalesce into large clusters.

grafted Bindzil particles, discrete gold particles are found almost exclusively in and around the melt of silica particles (see Fig. $2 \mathrm{~b}-\mathrm{d})$. This observation, together with the $-\mathrm{SH}$ peak found in the IR-spectrum of the MPTMS-treated Bindzil particles confirms the presence of thiol-groups on the silica surface. The gold particles vary in size, but we were not able to assess a clear relation between experimental conditions, such as the stirring and concentration of $\mathrm{NaBH}_{4}$ on the size or polydispersity of the gold particles. However, the amount of $\mathrm{HAuCl}_{4}$ was obtimized around $50 \mathrm{~mL}$ per gram Bindzil. When using more $\mathrm{HAuCl}_{4}$, free gold nanoparticles could be observed on the TEM-grid.

\subsubsection{Thiol-functionalization and gold adsorption onto PES-membranes covered with a silica crust}

PES membranes immersed in a Bindzil dispersion in ethanol, in particular the ones which had been soaked in ammonia solution prior to exposure to Bindzil (PESBiEt2) showed complete coverage of the polymer with silica nanoparticles, even inside the pores (see Fig. $3 \mathrm{c}$ and d). Membranes, which had not been soaked in ammonia first, had a less homogeneous coverage of Bindzil particles. Moreover, the bindzil particle crust remained intact even after exposure to ultrasonication, which suggests that attraction between silica and PES is unlikely to be due to electrostatic forces only. Deposition of Bindzil from water (PESBiW) resulted in clusters of Bindzil particles inside the pore-cavities rather than at the polymer surface. The PES-membranes, where silica growth had taken place directly onto the membranes (PESSt), were only partially covered with a silica crust as observed with SEM (see Fig. 4).

After thiol-functionalization and addition of gold particles, the color of the PESBiEt2 membrane had become homogeneously purple whereas the PESSt membrane was indeed purple, but the color was less homogeneous, which can be explained by the less homogeneous silica layer and therefore empty regions without SH-groups. In both cases, the color of the membrane remained the same after several days in continuously refreshed water. The TEM-picture in Fig. 5 shows the PESBiEt2 full of single gold particles attached to the Bindzil crust. 
(a)

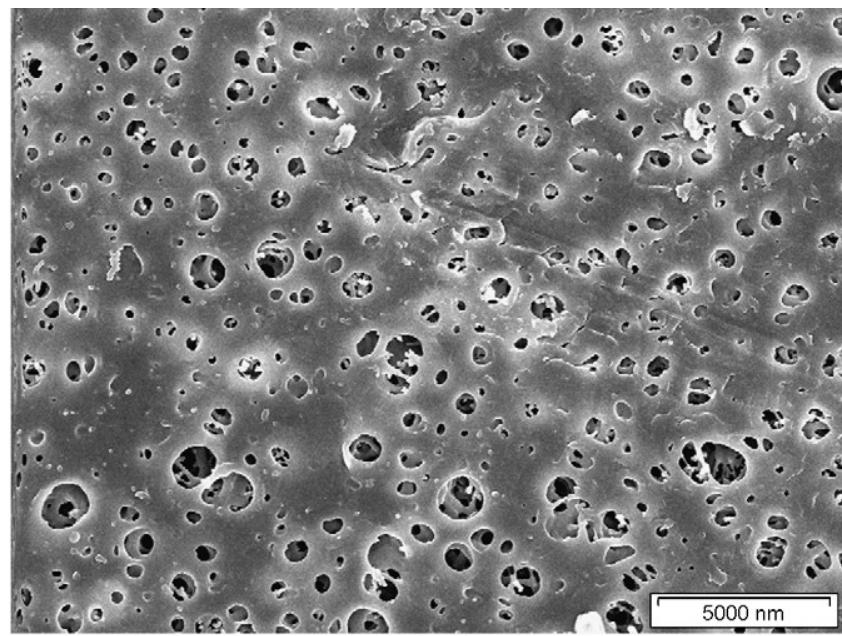

(c)

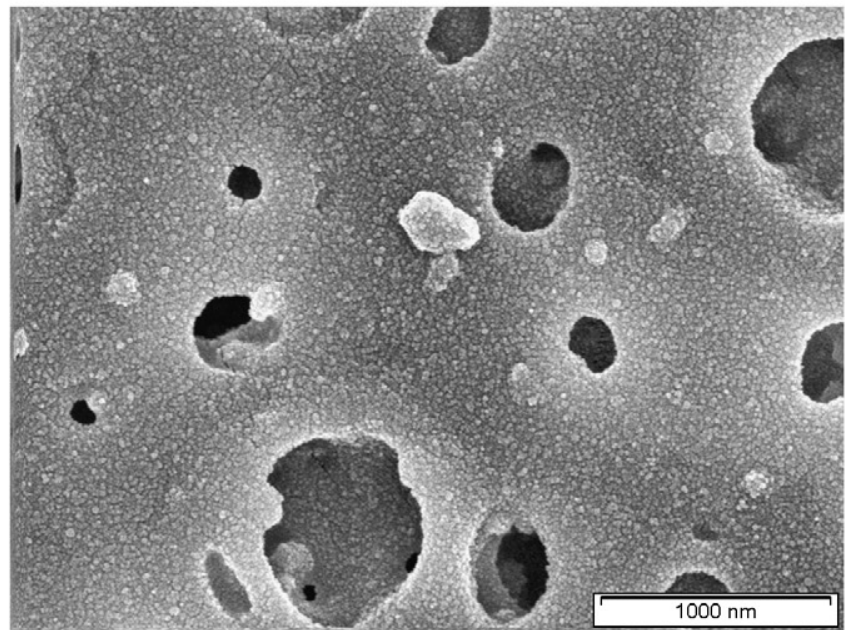

(b)

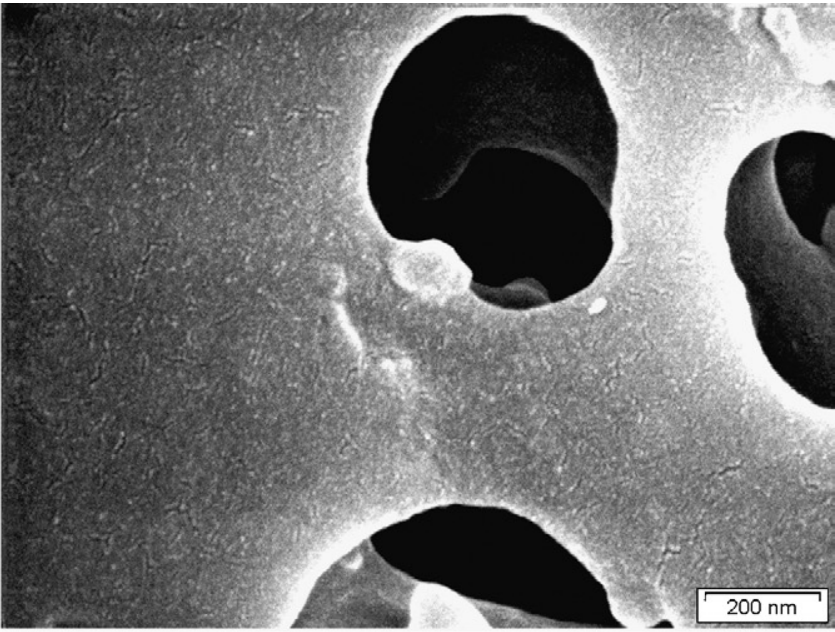

(d)

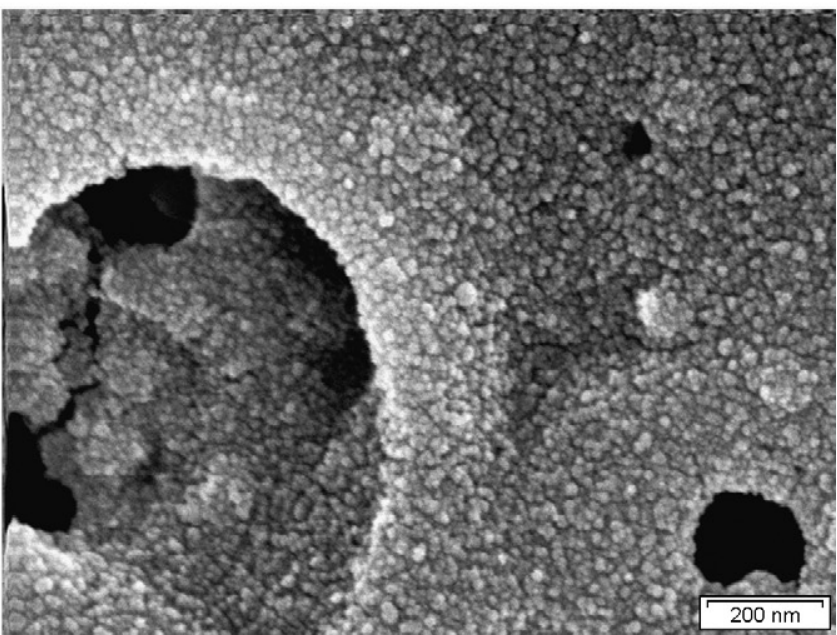

Fig. 3. SEM pictures of (a and b) PES-membranes as recieved, and (c and d) PES-membrane PESBiEt2 completely covered in a crust of Bindzil silica nanoparticles.

An important thing to note is that the reaction time for MPTMS with the silica covered filters had to be longer than for the other particles and grains mentioned in this paper. The fast method to let MPTMS react with the silica surface by par-

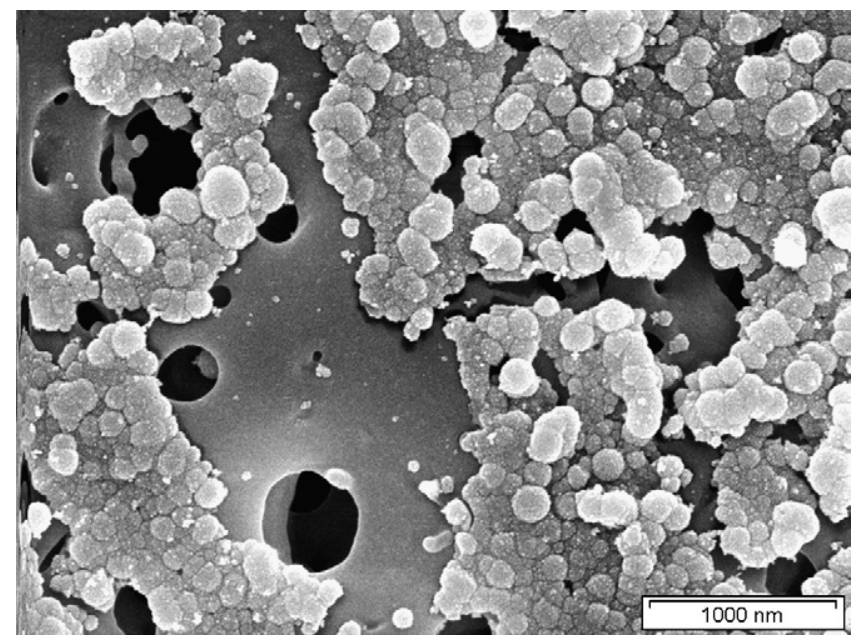

Fig. 4. SEM picture of PES-membrane PESSt. The membrane is only partially covered silica, which has been grown directly onto the membrane. tial removal of ammonia and solvent was not applicable for the filters.

\subsection{Thiol-functionalization and gold particle synthesis on Stöber silica}

The reduction of $\mathrm{HAuCl}_{4}$ in the presence of MPTMS-coated silica spheres resulted in silica spheres covered with gold particles with a diameter of 5-10 nm (see Fig. 6). We observed that using $0.1 \mathrm{M} \mathrm{NaBH}_{4}$ resulted in gold nanoparticles which were smaller and less polydisperse than when lowering the concentration of $\mathrm{NaBH}_{4}$ to $0.01 \mathrm{M}$.

After several cleaning steps, the homogeneous sediment of particles remained colored, in contrast to the sample with nonmodified silica, for which the sediment became white again as the free gold particles were removed. The silica-gold particles form colored, stable dispersions, which crystallize upon sedimentation. All dispersions remained stable for a long period of time and sedimented particles could easily be redispersed at least 6 months after preparation. Surprisingly, the gold covered silica particles can be coated with silica by using them as seeds in a Stöber synthesis (see Fig. 6), something which is not possible on pure gold surfaces, due to gold's low affin- 

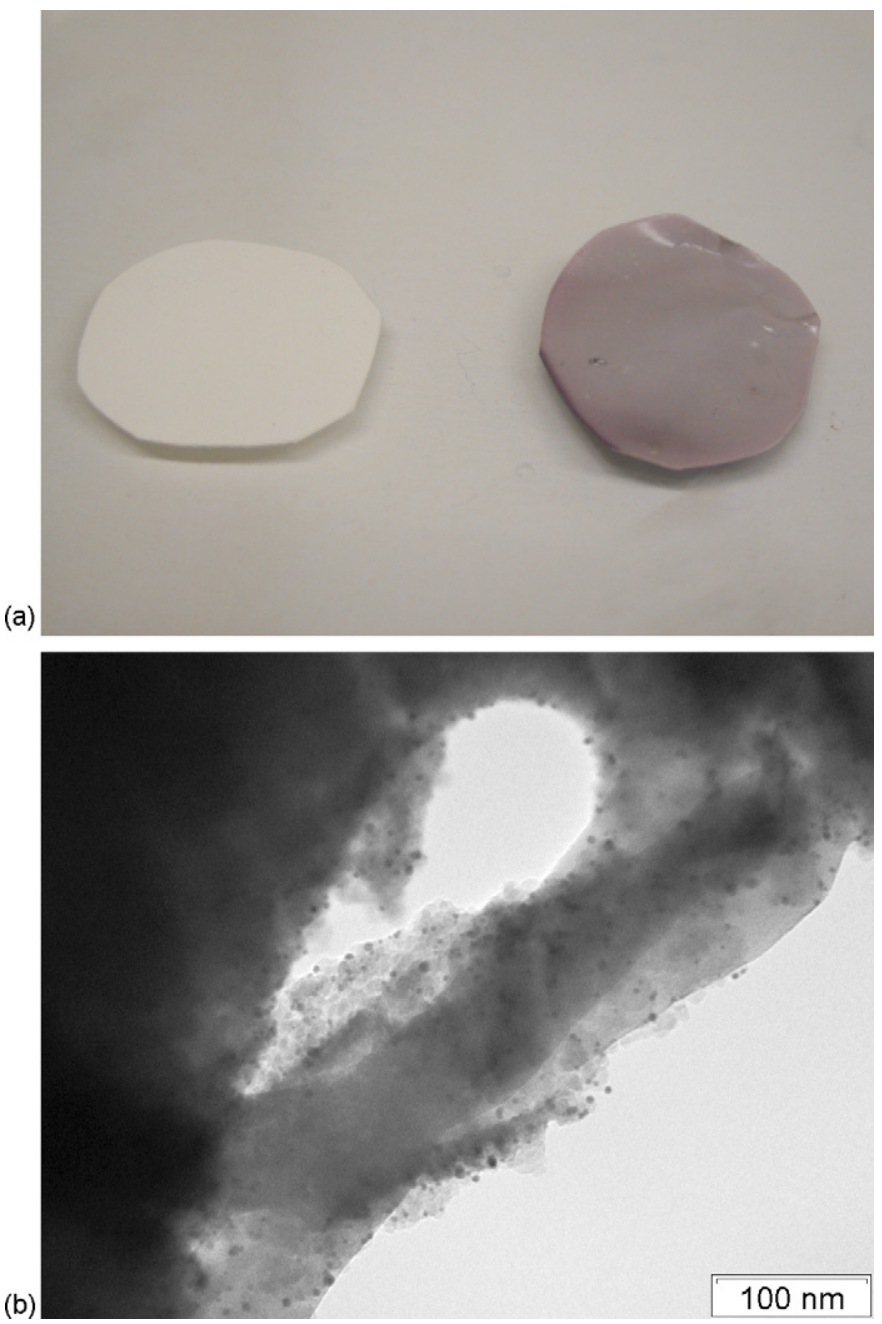

Fig. 5. (a) Photograph showing pieces of the thiol-functionalized Bindzilcovered PES-membrane before (left) and after (right) being soaked in a gold dispersion. (b) TEM-picture of the thiol-functionalized Bindzil-covered PES-membrane clearly showing the silica nanoparticles covering the polymer branches and the gold nanoparticles (higher contrast) attached to the silica.

ity for silica [26,27]. Very likely, newly formed silica nucleates on the silica surface in between the gold particles such that the gold particles are eventually capped by further deposition of silica.

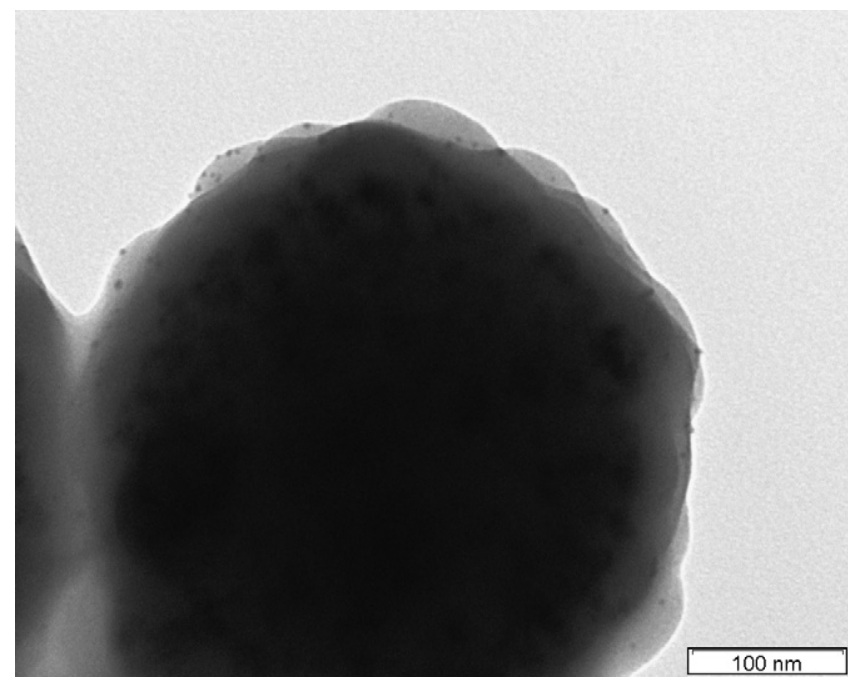

Fig. 7. TEM-picture of the magnetic silica particles (SibCs, $R=217 \mathrm{~nm}$ ), coated with MPTMS and covered with gold particles. The rough silica surface is due to the embedded cobalt ferrite particles, around which new silica has nucleated and grown.

We have previously shown that magnetic particles of cobalt ferrite and maghemite can be irreversibly attached to thiolfunctionalized silica particles and subsequently coated with a silica layer [15]. Here, it is shown how multi-layer-structure type of composite particles can be prepared, by functionalizing the outer silica shell of such magnetic silica colloids and perform the direct synthesis of gold nanoparticles. The resulting gold decorated magnetic silica particles are shown in Fig. 7.

\subsection{Thiol-functionalization and gold particle synthesis onto porous silica grains}

The procedure for grafting Stöber silica with MPTMS could be applied to porous glass grains as well as to SBA-15 without any additional modification. After synthesizing gold nanoparticles in the presence of the silica grains, and subsequent rinsing, a clear difference could be seen between the grains, which had been grafted with thiol groups, and the grains which had not. The thiol-functionalized grains remained colored, while the nonmodified grains became white again after rinsing with water.
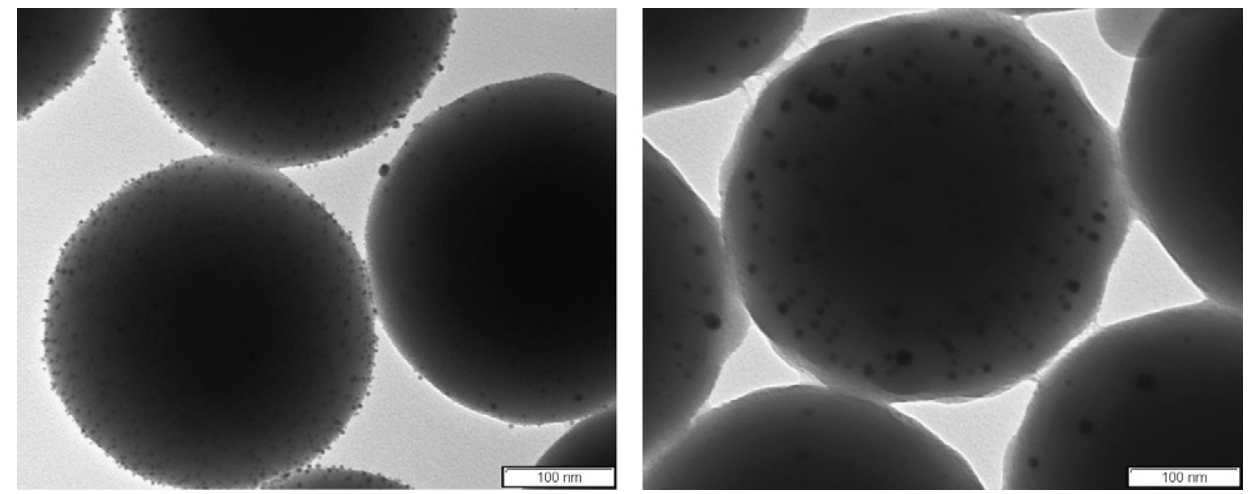

Fig. 6. TEM-pictures of silica-gold composite particles prepared via nucleation and growth of gold nanoparticles in the presence of thiol-functionalized Stöber silica (a) and after coating these composites with a $20 \mathrm{~nm}$ (b) silica layer, respectively. 
(a)
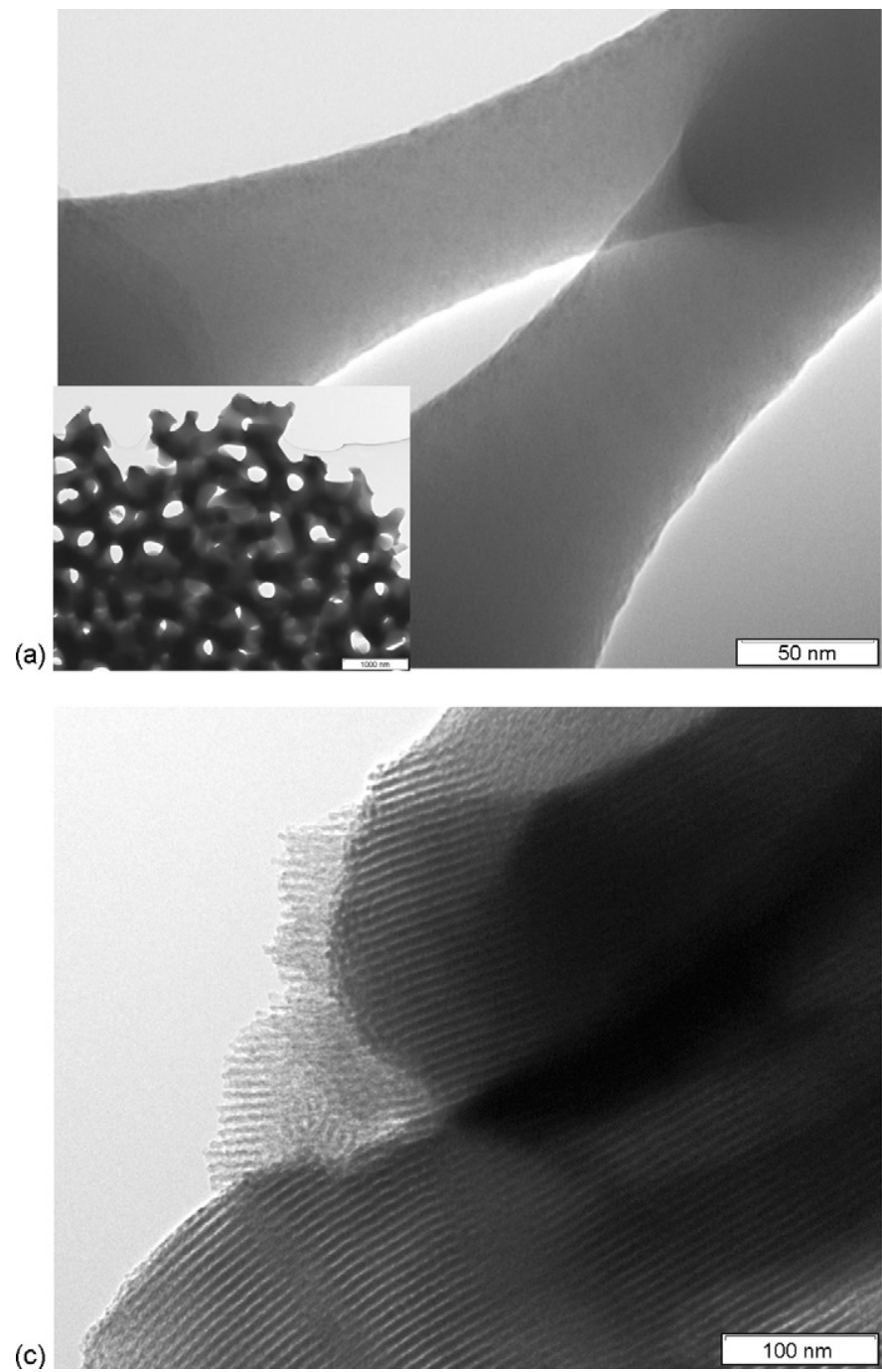

(b)

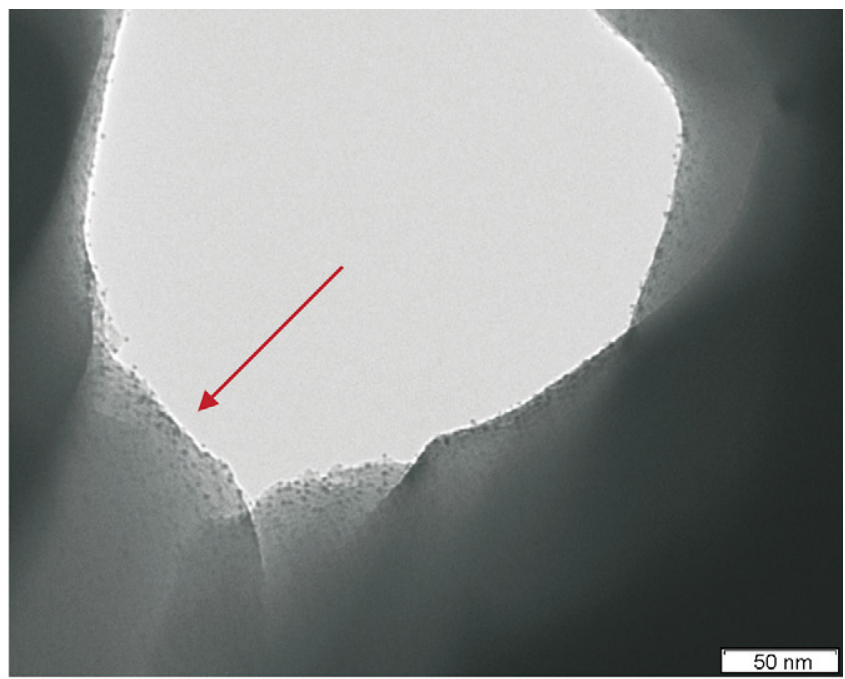

(d)

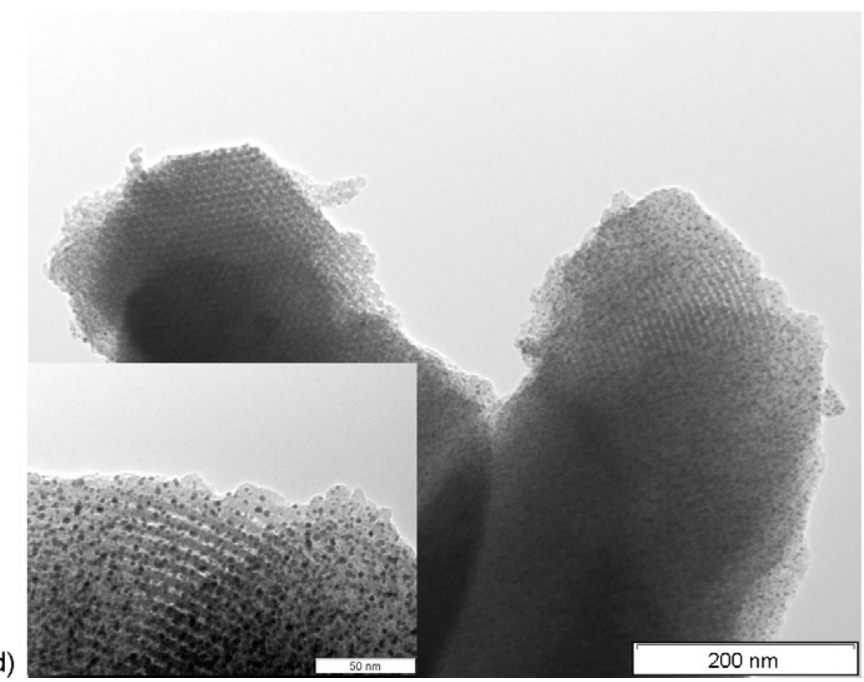

Fig. 8. TEM pictures showing the results of synthesis of gold particles in the presence of thiol-functionalized (a and b) and not thiol-functionalized (c and d) controlled porous glasses. In the presence of thiol groups, the surface of the pores are covered with small $(R \sim 1 \mathrm{~nm})$ gold particles.

TEM-pictures (see Fig. 8) show that also the surface of the pores in the modified CPG grains is covered with small $(R \sim 1-2 \mathrm{~nm})$ gold particles.

\section{Conclusions}

We have demonstrated that a method to functionalize silica colloids with thiol groups can be applied to a variety of silica particles and structures, including commercial silica nanoparticles and (magnetizable) Stöber silica as well as silica structures provided by porous glasses and novel polymer-templated silica membranes. Commercially available membranes of polyethersulfone can be encapsulated in a crust of silica, by addition of colloidal silica particles, which can form a dense layer around the polymer. The functionalization of silica particles and structures with thiol-groups allows for irreversible binding of metal(oxide) particles from a solution, as demonstrated here for gold as well as $\mathrm{CoFe}_{2} \mathrm{O}_{4}$ nanoparticles. The functionalization step generally includes transfer of the particles or grains to a mixture of ethanol and ammonia and addition of MPTMS followed by heating and removal of solvent for a fast reaction. For the smallest $(R \sim$ $6 \mathrm{~nm}$ ) silica particles, the grafting procedure must be slightly modified to avoid gelation. This includes purification from soluble silicates before transferring the particles from water to ethanol and thereafter not exceeding $\mathrm{pH} \sim 8.5$. Moreover, for the silica covered polymer membranes, longer reaction time is needed during the functionalization step. The reaction is in this case slowed down by lowering the temperature from 80 to $50{ }^{\circ} \mathrm{C}$ and not removing solvent. Gold nanoparticles can be synthesized directly in the presence onto the surface of the various types of functionalized silica in a convenient one-pot synthesis step to obtain a wide range of silica-gold composites. It is worth noticing here that the silica colloids remain stable in dispersion throughout this step, resulting in a dispersion of single golddecorated particles. Since these silica systems all have a large surface-to-volume ratio, surface modification of such materials with - SH groups and chemical adsorption of metals can be useful in for instance separation processes. 


\section{Acknowledgements}

Eka Chemicals are acknowledged for providing the Bindzil silica sols. Fuoad Sulimani is thanked for assistance with the IRmeasurements, Stefano Sacanna for making SEM-pictures and Johanna Schütz-Widoniak and Ana Ürmenyi for many helpful discussions. TEM-pictures were made at the EMU (Electron Microscopy Utrecht) facility. This research was financially supported by OCÉ Technologies, Venlo, The Netherlands.

\section{References}

[1] L. Chu, M.W. Daniels, L.F. Francis, Use of (glycidoxypropyl)trimethoxysilane as a binder in colloidal silica coatings, Chem. Mater. 9 (1997) 2577-2582.

[2] M. Zayat, D. Levy, Surface organic modifications and the performance of sol-gel derived gel-glass dispersed liquid crystals (gdlcs), Chem. Mater. 15 (2003) 2122-2128.

[3] C. Yang, H. Sheu, K. Chao, Templated synthesis and structural study of densely packed metal nanostructures in $\mathrm{mcm}-41$ and $\mathrm{mcm}-48$, Adv. Funct. Mater. 12 (2002) 143-148.

[4] C. Graf, A. van Blaaderen, Metallodielectric colloidal core-shell particles for photonic applications, Langmuir 18 (2002) 524-534.

[5] L. Arakaki, V.A. Filha, J. Espinola, M.d. Fonseca, S.d. Oliveira, T. Arakaki, C. Airoldi, New thiol adsorbent grafted on silica gel: synthesis, characterization and employment for heavy metal adsorptions, J. Environ. Monit. 5 (2003) 366-370.

[6] W. Stöber, A. Fink, E. Bohn, Controlled growth of monodisperse silica spheres in the micron size range, J. Colloid Interface Sci. 26 (1968) 62-69.

[7] A. Taguchi, F. Schüth, Ordered mesoporous materials in catalysis, Microporous Mesoporous Mater. 77 (2005) 1-45.

[8] S.G.J.M. Kluijtmans, A light scattering contrast variation of bicontinuous porous glass media, Langmuir 13 (1997) 4976-4981.

[9] B.J. Rappoli, D.A. Rowley, The sorption kinetics of copper(II) on chemically modified controlled pore glass, J. Colloid Interface Sci. 226 (2000) 218-221.

[10] A. Philipse, M.v. Bruggen, C. Pathmamanoharan, Magnetic silica dispersions: preparation and stability of surface modified silica particles with a magnetic core, Langmuir 10 (1994) 92-99.

[11] M.v. Bruggen, Preparation and properties of colloidal core-shell rods with adjustable aspect ratios, Langmuir 14 (1998) 2245-2255.
[12] C. Graf, D.L.J. Vossen, A. Imhof, A.v. Blaaderen, A general method to coat colloidal particles with silica, Langmuir 19 (2003) 6693-6700.

[13] E.P. Plueddemann, Silane Coupling Agents, Plenum Press, New York, 1982.

[14] A. Philipse, A. Vrij, Preparation and properties of nonaqueous model dispersions of chemically modified, charged silica spheres, J. Colloid Interface Sci. 128 (1) (1989) 121-136.

[15] E.M. Claesson, A. Philipse, Monodisperse magnetizable composite silica spheres with tunable dipolar interactions, Langmuir 21 (2005) 94129419.

[16] N. Hebalkar, S. Kharrazi, A. Ethiraj, J. Urban, R. Flink, S.K. Kulkarni, Structural and optical investigations of sio2-cds core-shell particles, J. Colloid Interface Sci. 278 (2004) 107-114.

[17] S.L. Wescott, S.J. Oldenburg, T.R. Lee, N.J. Halas, Formation and adsorption of clusters of gold nanoparticles onto funtionalized silica nanoparticle surfaces, Langmuir 14 (1998) 5396-5401.

[18] M.S. Fleming, D.R. Walt, Stability and exchange studies of alkanethiol monolayers on gold-nanoparticle-coated silica microspheres, Langmuir 17 (2001) 4836-4843.

[19] C. Bain, E. Troughton, Y.-T. Tao, J. Evall, G. Whitesides, R. Nuzzo, Formation of monolayer films by the spontaneous assembly of organic thiols from solution onto gold, J. Am. Ceram. Soc. 111 (1989) 321-335.

[20] M. Giersig, P. Mulvaney, Preparation of ordered colloid monolayers by electrophoretic deposition, Langmuir 9 (1993) 3408-3413.

[21] M.L. Anderson, C.A. Morris, R.M. Stroud, C.I. Merzbacher, D.R. Rolison, Colloidal gold aerogels: properties, and characterization, Langmuir 15 (1999) 674-681.

[22] G. H. Bogush, M. A. Tracy, C. F. Z. IV, Preparation of monodisperse silica particles: control of size and massfraction, J. Non-Cryst. Solids 104 (1988) 95-106.

[23] A. Urmenyi, A. Philipse, R. Lammertink, M. Wessling, Polymer-in-a-crust membranes: macroporous materials with tunable surface functionality, Langmuir 22 (2006) 5459-5468.

[24] C. Pathmamanoharan, A. Philipse, Preparation of small alkane-grafted silica particles (for saxs and sans studies) from aqueous commersial sols, J. Colloid Interface Sci. 165 (1994) 519-521.

[25] A.P. Philipse, Particulate colloids: aspects of preparation and characterization, in: J. Lycklema (Ed.), Fundamentals of Interface and Colloid Science, vol. IV, Academic Press, 2005.

[26] L.M. Liz-Marzán, A.P. Philipse, Synthesis and optical properties of goldlabeled silica particles, J. Colloid Interface Sci. 176 (1995) 459-466.

[27] L.M. Liz-Marzán, M. Giersig, P. Mulvaney, Synthesis of nanosized goldsilica core-shell particles, Langmuir 12 (1996) 4329-4335. 